\title{
T2-Weighted Dixon Turbo Spin Echo for Accelerated Simultaneous Grading of Whole-Body Skeletal Muscle Fat Infiltration and Edema in Patients With Neuromuscular Diseases
}

\author{
Sarah Schlaeger, ${ }^{*}+$ Elisabeth Klupp, MD, * Dominik Weidlich, MSc, $\dagger$ Barbara Cervantes, MSc, $\dagger$ \\ Sarah C. Foreman, * Marcus Deschauer, MD, $\neq$ Benedikt Schoser, MD, $\$$ Christoph Katemann,// \\ Hendrik Kooijman, PhD,// Ernst J. Rummeny, MD, † Claus Zimmer, MD, * \\ Jan S. Kirschke, $P D,{ }^{*}$ and Dimitrios C. Karampinos, $P D^{\dagger}$
}

\begin{abstract}
Objective: The assessment of fatty infiltration and edema in the musculature of patients with neuromuscular diseases (NMDs) typically requires the separate performance of $\mathrm{T}_{1}$-weighted and fat-suppressed $\mathrm{T}_{2}$-weighted sequences. $\mathrm{T}_{2}$-weighted Dixon turbo spin echo (TSE) enables the generation of $\mathrm{T}_{2}$-weighted fat- and water-separated images, which can be used to assess both pathologies simultaneously. The present study examines the diagnostic performance of $\mathrm{T}_{2}$-weighted Dixon TSE compared with the standard sequences in 10 patients with NMDs and 10 healthy subjects.

Methods: Whole-body magnetic resonance imaging was performed including $\mathrm{T}_{1}$-weighted Dixon fast field echo, $\mathrm{T}_{2}$-weighted short-tau inversion recovery, and $\mathrm{T}_{2}$-weighted Dixon TSE. Fatty infiltration and intramuscular edema were rated by 2 radiologists using visual semiquantitative rating scales. To assess intermethod and interrater agreement, weighted Cohen's $\kappa$ coefficients were calculated.

Results: The ratings of fatty infiltration showed high intermethod and high interrater agreement $\left(\mathrm{T}_{1}\right.$-weighted Dixon fast field echo vs $\mathrm{T}_{2}$-weighted Dixon TSE fat image). The evaluation of edematous changes showed high intermethod and good interrater agreement $\left(\mathrm{T}_{2}\right.$-weighted short-tau inversion recovery vs $\mathrm{T}_{2}$-weighted Dixon TSE water image).

Conclusions: $T_{2}$-weighted Dixon TSE imaging is an alternative for accelerated simultaneous grading of whole-body skeletal muscle fat infiltration and edema in patients with NMDs.
\end{abstract}

Key Words: skeletal muscle, fat infiltration, edema, neuromuscular diseases, Dixon MRI

(J Comput Assist Tomogr 2018;42: 574-579)

A cute inflammatory edematous alterations of skeletal muscles reflecting disease activity as well as fatty infiltration of chronically affected muscles are two important characteristic changes that can be detected by imaging in neuromuscular diseases (NMDs). ${ }^{1-3}$ Magnetic resonance imaging (MRI) has been emerging as a popular imaging technique in the diagnostic procedure of NMD patients to characterize whole-body disease progression as well as to guide muscle biopsy sites. ${ }^{4}$ For the detection of qualitative pathological changes in the muscle tissue, $\mathrm{T}_{1}$-weighted and fat-suppressed

From the Departments of *Diagnostic and Interventional Neuroradiology, $\dagger$ Diagnostic and Interventional Radiology, and \$Neurology, Technical University of Munich; §Friedrich-Baur-Institut, Ludwig Maximilian University, Munich; and ||Philips Healthcare, Hamburg, Germany.

Received for publication October 2, 2017; accepted December 26, 2017.

Correspondence to: Sarah Schlaeger, Department of Diagnostic and

Interventional Neuroradiology, Klinikum rechts der Isar, Ismaninger Str 22, 81675 Munich, Germany (e-mail: sarah.schlaeger@tum.de).

S.S and E.K. contributed equally to this work.

The authors declare no conflict of interest.

Copyright (C) 2018 Wolters Kluwer Health, Inc. All rights reserved.

DOI: $10.1097 /$ RCT.0000000000000723
$\mathrm{T}_{2}$-weighted sequences have traditionally been used..$^{5}$ To overcome the sensitivity of chemical-selective fat-suppression techniques to off-resonance effects, short-tau inversion recovery (STIR) turbo spin echo (TSE) sequences have been used to characterize $T_{2}$ hyperintensities. For the classification of the extent of fatty infiltration as well as of edematous muscular alterations, semiquantitative rating scales based on conventional MR images are commonly used. The Mercuri scale is standardly used to categorize the fatty infiltration of muscle tissue starting from $0 \%$ (score 1 ) to above $60 \%$ (score 4 ) on axial $\mathrm{T}_{1}$-weighted images. ${ }^{6}$ To categorize intramuscular $\mathrm{T}_{2}$ hyperintensities indicating potential muscle edema, the 3-point rating Morrow scale can be applied on fat-suppressed $\mathrm{T}_{2}$-weighted images. ${ }^{7}$

To evaluate fatty infiltration and edematous changes in the muscle tissue precisely, a whole-body MRI is needed for the assessment of both $\mathrm{T}_{1}$-weighted and STIR sequences. The acquisition of these 2 sequences in a whole-body scan protocol can lengthen the total acquisition time, as at least 5 stacks are needed to cover the whole body and because of the necessity to perform additional prescans per stack. A longer scan time can be problematic particularly in severely affected patients suffering from pain or from diaphragm weakness causing respiratory problems in the laying position. In cases when longer acquisition times cannot be tolerated, it would be advantageous to have a single sequence enabling the same diagnostic accuracy as the conventional $\mathrm{T}_{1}$-weighted and STIR methods for simultaneous assessment of fatty infiltration and intramuscular edema in a shorter acquisition time. The $\mathrm{T}_{2}$-weighted Dixon TSE sequence enables the simultaneous generation of $\mathrm{T}_{2}$-weighted fat-separated and water-separated fat suppressed images, ${ }^{8,9}$ allowing the simultaneous assessment of fatty and edematous alterations.

The purpose of the present work is to examine and compare the diagnostic accuracy of typical $\mathrm{T}_{1}$-weighted and STIR MRI sequences with the proposed $\mathrm{T}_{2}$-weighted Dixon TSE for accelerated simultaneous grading of whole-body skeletal muscle fat infiltration as well as edema in patients with different NMDs and healthy subjects.

\section{MATERIALS AND METHODS}

\section{Subjects}

Ten patients ( 6 females and 4 males; $48.1 \pm 9.3$ years) with varied mainly hereditary NMDs were recruited, including myotonic dystrophy type $2(\mathrm{DM} 2)(\mathrm{n}=4)$, limb girdle muscular dystrophy type 2A (LGMD2A) $(\mathrm{n}=2)$, limb girdle muscular dystrophy type 2I (LGMD2I) ( $=1)$, Becker muscular dystrophy (BMD) $(\mathrm{n}=1)$, amyotrophic lateral sclerosis $(\mathrm{n}=1)$, and centronuclear 
myopathy $(\mathrm{CNM})(\mathrm{n}=1)$. Diagnosis based on muscle biopsies and/or genetic testing. In addition, 10 healthy volunteers ( 7 females and 3 males; $43.7 \pm 19.7$ years) were recruited and served as control group. These volunteers had no history of neuromuscular disorders or other neurological diseases, previous muscle injury, and MRI contraindication. The study was approved by the ethics commission of the medical faculty of the Technical University of Munich. Before participation, all subjects gave written informed consent.

\section{MRI Measurements}

All 20 subjects underwent whole-body MRI on a 3T system (Ingenia, Philips Healthcare, Best, Netherlands) using a wholebody coil, the built-in 12-channel posterior coil and a 16-channel anterior coil, which was placed on top of the hip and thigh region to ensure best signal quality for these muscle groups. Subjects were positioned in a head-first supine position. Three groups of pulse sequences were performed in 5 axial stacks starting from the neck region to the talocalcanean joint. Pulse sequences included a 2-echo 3-dimensional $\mathrm{T}_{1}$-weighted Dixon fast field echo (FFE) (TR/TE1/ TE2, 2.8/0.9/1.7 ms; field of view (FOV), $450 \times 330 \times 306 \mathrm{~mm}^{3}$; acquisition voxel size, $2.5 \times 2.7 \times 6.0 \mathrm{~mm}^{3}$; slices, 51; averages, 1 ), a 2-dimensional $\mathrm{T}_{2}$-weighted STIR TSE (TR/TE, 4620/100 ms;
FOV, $330 \times 450 \times 306 \mathrm{~mm}^{3}$; inversion recovery delay, $240 \mathrm{~ms}$; acquisition voxel size, $2.5 \times 2.7 \times 6.0 \mathrm{~mm}^{3}$; slices, 26 ; slice gap, $6 \mathrm{~mm}$; TSE factor, 41; averages, 2), and a 2-echo 2-dimensional Dixon TSE to simultaneously generate $\mathrm{T}_{2}$-weighted fat- and water-separated images (TR/TE/ $\Delta \mathrm{TE}, 3725 / 100 / 1.0 \mathrm{~ms}$; FOV, $330 \times 450 \times 306 \mathrm{~mm}^{3}$; acquisition voxel size, $2.5 \times 2.7 \times 6.0 \mathrm{~mm}^{3}$; slices, 26; slice gap, $6 \mathrm{~mm}$; TSE factor, 45; averages, 2). Water-fat separation of the TSE and FFE images was performed online using the vendor's mDixon algorithm. ${ }^{8}$ Acquisition of the Dixon FFE sequence required a single breath-hold for the 3 upper body stacks. Total scan time for the 3 sequences was approximately 45 minutes (scan time per stack: T1W Dixon FFE, 12 s; T2W STIR TSE, 139 s; T2W Dixon TSE, 127 s).

\section{Semiquantitative Assessment}

To compare the diagnostic performance of the 3 sequences, 2 different visual semiquantitative rating scales were used to evaluate the degree of pathological development in the muscle tissue, including fatty infiltration and intramuscular edema. To assess interreader agreement, the images were evaluated independently by 2 readers: a radiologist (E. K., reader 1 ) with 2.5 years of experience in muscle imaging and a radiologist (J.S.K., reader 2) with

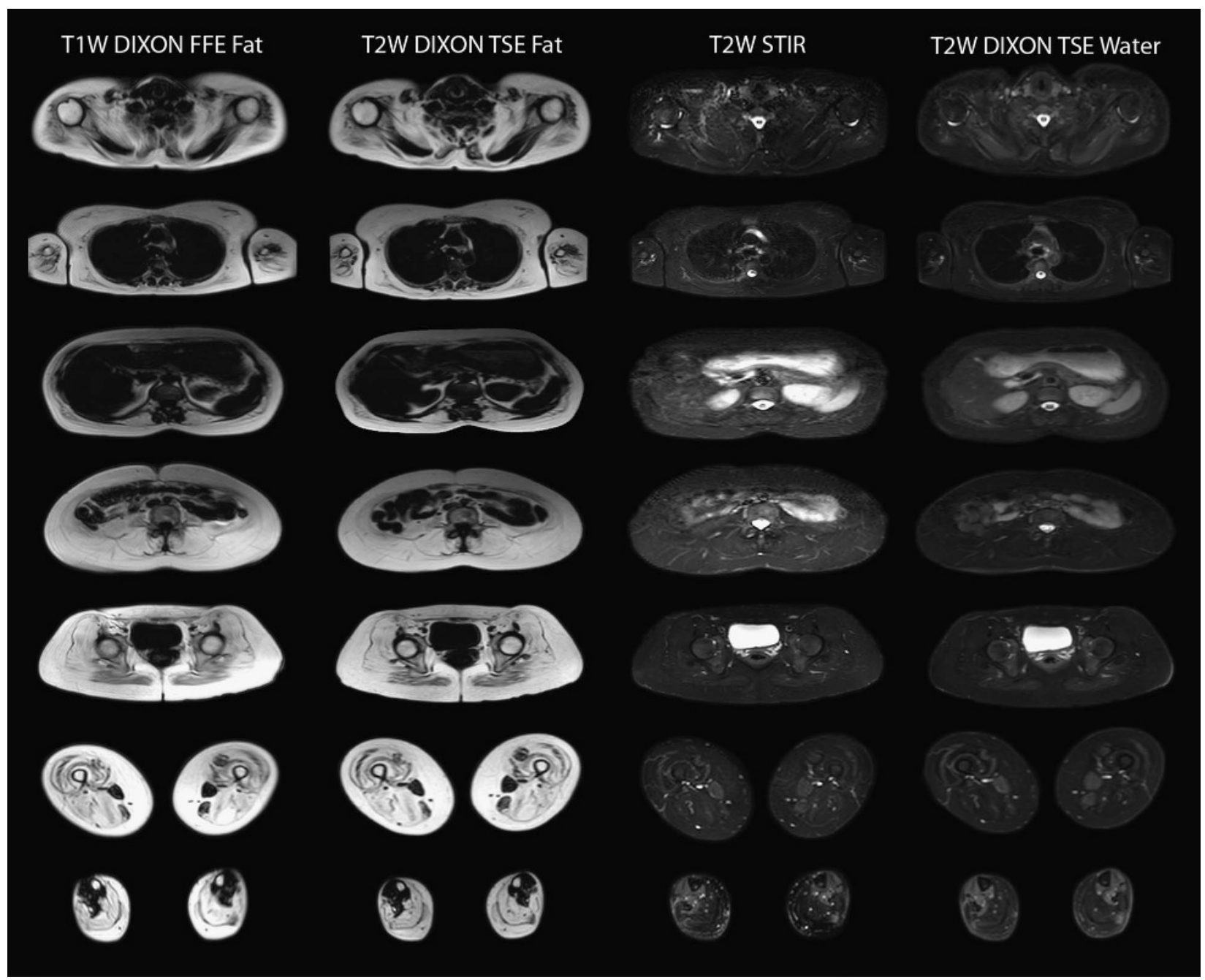

FIGURE 1. Whole-body Dixon FFE fat-separated, $T_{2}$-weighted Dixon TSE fat-separated, $T_{2}$-weighted STIR, and $T_{2}$-weighted Dixon TSE water-separated images in a severely affected LGMD2A patient. 
10 years of experience in muscle imaging. To avoid any bias, the ratings of each reader were performed blindly in a randomized order.

Severity of fatty infiltration was evaluated via the four point Mercuri grading scale ${ }^{6}$ with score 1 for muscles with normal appearance, score 2 for muscles with mild fatty infiltration (less than $30 \%$ of the volume of the individual muscle), score 3 for muscles with moderate fatty infiltration $(30 \%$ to $60 \%$ of the volume of the individual muscle), and score 4 for muscles with severe fatty infiltration (more than $60 \%$ of the volume of the individual muscle). The Mercuri grading scale was applied independently on Dixon FFE fat and $\mathrm{T}_{2}$-weighted Dixon TSE fat images.

To rate intramuscular hyperintensities indicating edema, a 3-point scale published by Morrow et $\mathrm{al}^{7}$ was used with score 0 for absence of intramuscular hyperintensity, score 1 for mild hyperintensity, and score 2 for definite intramuscular hyperintensity. The Morrow grading scale was applied independently on $\mathrm{T}_{2}$-weighted STIR images and $\mathrm{T}_{2}$-weighted Dixon TSE water images.

A total of 24 muscles were scored bilaterally in each subject to cover different regions of the entire body: subscapularis and pectoralis major (shoulder region); thoracal and lumbar part of the multifidus muscle, longissimus thoracis, and iliocostalis lumborum (paraspinal region); gluteus maximus, gluteus medius, and gluteus minimus (hip region); rectus femoris, vastus lateralis, vastus medialis, vastus intermedius, sartorius, gracilis, adductor longus, adductor magnus, adductor brevis, semitendinosus, semimembranosus, short and long head of the biceps femoris (thigh region); tibialis anterior and gastrocnemius medialis (lower leg region). Muscles that could not be rated due to artifacts or premature abortion of the scan by the patient and therefore needed to be excluded from further analyses were documented.

\section{Statistical Analysis}

The Mercuri scores evaluated on the $\mathrm{T}_{1}$-weighted Dixon FFE were compared with the Mercuri scores evaluated on the $T_{2}$-weighted Dixon TSE fat images. The Morrow scores evaluated on the $\mathrm{T}_{2}$-weighted STIR TSE were compared with the Morrow scores on the $T_{2}$-weighted Dixon TSE water images. To quantitatively assess the intermethod agreement of these different sequences, linearly weighted Cohen's $\kappa$ coefficients ${ }^{10}$ were calculated. To quantitatively assess the interrater agreement of the 2 readers, linearly weighted Cohen's $\kappa$ coefficients were used as well. The

TABLE 1. Sequence Agreement on Fatty Infiltration Grading Between Dixon FFE Fat and $T_{2}$-Weighted Dixon TSE Fat Images in Patients by Reader 1 and by Reader 2

\section{T2W Dixon TSE Fat}

\begin{tabular}{lrrrrr}
\hline Reader 1 & $\mathbf{1}$ & $\mathbf{2}$ & $\mathbf{3}$ & $\mathbf{4}$ & Total \\
T1W Dixon FFE fat & & & & \\
$\mathbf{1}$ & 186 & 13 & 0 & 0 & 199 \\
$\mathbf{2}$ & 8 & 75 & 4 & 0 & 87 \\
$\mathbf{3}$ & 2 & 8 & 62 & 0 & 72 \\
$\mathbf{4}$ & 0 & 0 & 4 & 103 & 107 \\
Total & 196 & 96 & 70 & 103 & 465 \\
\hline Reader 2 & $\mathbf{1}$ & $\mathbf{2}$ & $\mathbf{3}$ & $\mathbf{4}$ & Total \\
T1W Dixon & FFE fat & & & & \\
$\mathbf{1}$ & 133 & 22 & 2 & 2 & 159 \\
$\mathbf{2}$ & 54 & 82 & 12 & 6 & 154 \\
$\mathbf{3}$ & 0 & 13 & 36 & 16 & 65 \\
$\mathbf{4}$ & 0 & 2 & 8 & 77 & 87 \\
Total & 187 & 119 & 58 & 101 & 465 \\
\hline
\end{tabular}

TABLE 2. Sequence Agreement on Fatty Infiltration Grading between Dixon FFE Fat and $T_{2}$-Weighted Dixon TSE Fat Images in healthy Volunteers by Reader 1 and by Reader 2

\begin{tabular}{lrrrrr}
\hline \multicolumn{5}{c}{ T2W Dixon TSE Fat } & \\
\hline Reader 1 & $\mathbf{1}$ & $\mathbf{2}$ & $\mathbf{3}$ & $\mathbf{4}$ & Total \\
T1W Dixon FFE fat & & & & & \\
$\quad \mathbf{1}$ & 478 & 2 & 0 & 0 & 480 \\
$\mathbf{2}$ & 0 & 0 & 0 & 0 & 0 \\
$\mathbf{3}$ & 0 & 0 & 0 & 0 & 0 \\
$\mathbf{4}$ & 0 & 0 & 0 & 0 & 0 \\
Total & 478 & 2 & 0 & 0 & 480 \\
\hline Reader 2 & $\mathbf{1}$ & $\mathbf{2}$ & $\mathbf{3}$ & $\mathbf{4}$ & Total \\
T1W Dixon FFE fat & & & & & \\
$\mathbf{1}$ & 429 & 17 & 0 & 0 & 446 \\
$\mathbf{2}$ & 2 & 32 & 0 & 0 & 34 \\
$\mathbf{3}$ & 0 & 0 & 0 & 0 & 0 \\
$\mathbf{4}$ & 0 & 0 & 0 & 0 & 0 \\
Total & 431 & 49 & 0 & 0 & 480 \\
\hline
\end{tabular}

Cohen's $\kappa$ coefficient is a statistical parameter that is less than or equal to 0 when the agreement is indistinguishable from a random agreement and equal to 1 in case of perfect match. The recommended interpretation of the $\kappa$ values between 0 and 1 is as follows: below 0.20 is a poor agreement; 0.21 to 0.40 , a fair agreement; 0.41 to 0.60 , a moderate agreement; 0.61 to 0.80 , a substantial agreement; and above 0.81 , an almost perfect agreement. ${ }^{10}$ Thus, Cohen's $\kappa$ is more robust than simple percent agreement. The weighted version of Cohen's $\kappa$ coefficient is suggested when ordered items are compared, because it weights larger differences between scores stronger than small differences.

\section{RESULTS}

\section{Whole-Body MRI}

Figure 1 shows representative axial images of a severely affected LGMD2A patient obtained with the Dixon FFE, $\mathrm{T}_{2}$-weighted Dixon TSE (fat-separated), $\mathrm{T}_{2}$-weighted STIR, and $\mathrm{T}_{2}$-weighted Dixon TSE (water-separated) methods. A purely qualitative visual comparison of these sequences shows the similar diagnostic performance of $\mathrm{T}_{1}$-weighted Dixon FFE fat versus $\mathrm{T}_{2}$-weighted Dixon TSE fat images as well as $\mathrm{T}_{2}$-weighted STIR TSE versus $\mathrm{T}_{2}$-weighted Dixon TSE water images regarding the detection of fatty infiltration and edematous changes, respectively. In the shoulder region in particular, $\mathrm{T}_{2}$-weighted Dixon TSE seems to have a better image quality due to a better SNR and less artifacts. In general, concerning all patients and volunteers ( 20 in total), not all muscles could be evaluated and included in the analyses due to premature abortion of the examination by the patient. In 6 out of the 20 scanned subjects muscles were excluded. A total of 945 of 960 (ratio, 98.4\%) muscles for fatty infiltration and 949 of 960 muscles (ratio, 98.9\%) for intramuscular edema were scored.

\section{Grading of Fatty Infiltration}

Table 1 summarizes the Mercuri scores based on the $T_{1}$-weighted Dixon FFE fat and the $\mathrm{T}_{2}$-weighted Dixon TSE fat images for the 10 patients. The fatty infiltration ratings of the first reader resulted in a weighted $\kappa$ score of 0.93 , and the fatty infiltration ratings of the second reader yielded a weighted $\kappa$ score of 0.74 . Table 2 summarizes the Mercuri scores based on the $T_{1}$-weighted Dixon FFE fat and the $\mathrm{T}_{2}$-weighted Dixon TSE fat images for the 10 healthy 
volunteers. The weighted $\kappa$ value for interrater agreement on grading of fatty infiltration was 0.74 on $\mathrm{T}_{1}$-weighted Dixon FFE and 0.77 on $\mathrm{T}_{2}$-weighted Dixon TSE fat images including all rated muscles in patients and healthy volunteers (945 muscles in total).

Figure 2 shows the Mercuri grading results in a patient's shoulder region (LGMD2I), a patient's paraspinal region (BMD), and a patient's thigh region (BMD). Affected muscles were graded equally on both $\mathrm{T}_{1}$-weighted Dixon FFE fat and $\mathrm{T}_{2}$-weighted Dixon TSE fat images: the pectoralis major, the multifidus, and the gracilis with score 1; the longissimus thoracis and the sartorius with score 2; the subscapularis and the vastus lateralis with score 3; and the iliocostalis lumborum and the long head of the biceps femoris with score 4.

\section{Grading of Edematous Changes}

Table 3 summarizes the Morrow scores based on the $\mathrm{T}_{2}$-weighted STIR and the $\mathrm{T}_{2}$-weighted Dixon TSE water images for the 10 patients. The edematous changes ratings of the first reader resulted in a weighted $\kappa$ score of 0.77 , and the edematous changes ratings of the second reader resulted in a weighted $\kappa$ score of 0.80 . Table 4 summarizes the Morrow scores based on the $\mathrm{T}_{2}$-weighted STIR TSE and the $\mathrm{T}_{2}$-weighted Dixon TSE water images for the 10 healthy volunteers. Considering all muscles in all subjects, the weighted $\kappa$ value for interrater agreement on grading of edematous changes was 0.68 on $\mathrm{T}_{2}$-weighted STIR and 0.58 on $\mathrm{T}_{2}$-weighted Dixon TSE water images (949 muscles in total).

Figure 3 shows the Morrow grading in a patient's shoulder region (DM2), a patient's thigh region (BMD), and a patient's lower leg region (CNM). Affected muscles were graded equally on both the $\mathrm{T}_{2}$-weighted STIR and the $\mathrm{T}_{2}$-weighted Dixon TSE water images: the subscapularis and tibialis anterior with score 0 , the subscapularis and gastrocnemius medialis score 1, and the semimembranosus with score 2 .

\section{DISCUSSION}

The present study shows a high intermethod agreement between $\mathrm{T}_{1}$-weighted Dixon FFE and $\mathrm{T}_{2}$-weighted Dixon TSE fat images for rating fatty infiltration of muscles as well as between
TABLE 3. Sequence Agreement on Edema Grading Between $\mathrm{T}_{2}$-Weighted STIR and $\mathrm{T}_{2}$-Weighted Dixon TSE Water Images in Patients by Reader 1 and by Reader 2

\begin{tabular}{lrrrr}
\hline & \multicolumn{4}{c}{ T2W Dixon TSE Water } \\
\hline Reader 1 & $\mathbf{0}$ & $\mathbf{1}$ & $\mathbf{2}$ & Total \\
T2W STIR TSE & & & & \\
$\quad \mathbf{0}$ & 392 & 11 & 2 & 405 \\
$\mathbf{1}$ & 10 & 35 & 4 & 49 \\
$\mathbf{2}$ & 0 & 5 & 11 & 16 \\
$\quad$ Total & 402 & 51 & 17 & 470 \\
\hline Reader 2 & $\mathbf{0}$ & $\mathbf{1}$ & $\mathbf{2}$ & Total \\
T2W STIR TSE & & & & \\
$\mathbf{0}$ & 376 & 5 & 0 & 381 \\
$\mathbf{1}$ & 15 & 10 & 1 & 26 \\
$\mathbf{2}$ & 10 & 5 & 48 & 63 \\
Total & 401 & 20 & 49 & 470 \\
\hline
\end{tabular}

$\mathrm{T}_{2}$-weighted STIR and $\mathrm{T}_{2}$-weighted Dixon TSE water images for rating intramuscular edematous changes in patients with different NMDs and healthy subjects, respectively. Thus, $\mathrm{T}_{2}$-weighted Dixon TSE imaging should be considered as an alternative for accelerated simultaneous grading of whole-body skeletal muscle fat infiltration and edema.

The $\mathrm{T}_{2}$-weighted Dixon TSE approach shows a number of advantages compared with the acquisition of separate $T_{1}$-weighted and $\mathrm{T}_{2}$-weighted STIR scans. First, the $\mathrm{T}_{2}$-weighted Dixon sequence (127 s scan time per stack) allows the simultaneous separation of water and fat images in a shorter scan time than the sequential $\mathrm{T}_{1}$-weighted (12 s scan time per stack) and $\mathrm{T}_{2}$-weighted STIR acquisitions (139 s scan time per stack). ${ }^{8,9}$ The scan time reduction of 24 seconds per stack leads to a total scan time reduction of 120 seconds when performing 5 stacks to cover the whole body; thus, the resulting scan time reduction associated with the $\mathrm{T}_{2}$-weighted Dixon is especially beneficial when performing time-consuming whole-body MRI. In addition, the necessity

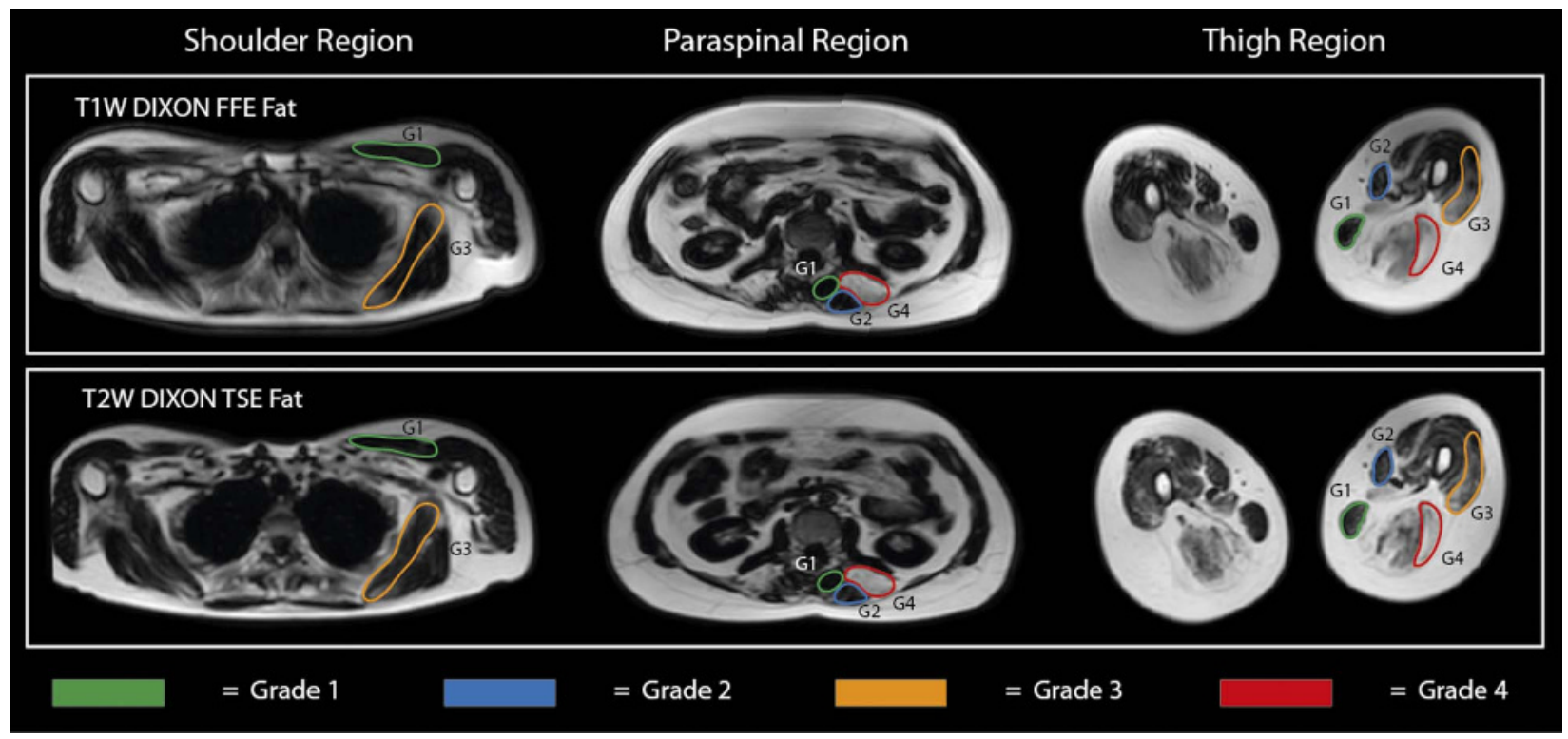

FIGURE 2. Mercuri grading of fatty infiltration based on Dixon FFE fat and $T_{2}$-weighted Dixon TSE fat images in a patient's shoulder region (LGMD2I), a patient's paraspinal region (BMD), and a patient's thigh region (BMD). Figure 2 can be viewed online in color at www.jcat.org. 
TABLE 4. Sequence Agreement on Edema Grading Between $\mathrm{T}_{2}$-Weighted STIR and T2-Weighted Dixon TSE Water Images in Healthy Volunteers by Reader 1 and by Reader 2

\begin{tabular}{lrrrr}
\hline & \multicolumn{4}{c}{ T2W Dixon TSE water } \\
\hline $\begin{array}{l}\text { Reader 1 } \\
\text { T2W STIR TSE }\end{array}$ & $\mathbf{0}$ & $\mathbf{1}$ & $\mathbf{2}$ & Total \\
$\quad \mathbf{0}$ & 469 & 1 & 0 & 470 \\
$\mathbf{1}$ & 0 & 6 & 0 & 6 \\
$\mathbf{2}$ & 0 & 0 & 3 & 3 \\
$\quad$ Total & 469 & 7 & 3 & 479 \\
\hline Reader 2 & $\mathbf{0}$ & $\mathbf{1}$ & $\mathbf{2}$ & Total \\
T2W STIR TSE & & & & \\
$\quad$ 0 & 439 & 11 & 0 & 450 \\
$\mathbf{1}$ & 9 & 16 & 1 & 26 \\
$\mathbf{2}$ & 0 & 2 & 1 & 3 \\
Total & 448 & 29 & 2 & 479 \\
\hline
\end{tabular}

to perform two different sequences to assess edematous and fatty changes requires the scan of two prescans for each sequence per stack, whereas the single $\mathrm{T}_{2}$-weighted Dixon TSE only requires one prescan per stack. The reduction in prescans leads to a further reduction in scan time when using the $\mathrm{T}_{2}$-weighted Dixon TSE sequence. Second, the presently implemented $\mathrm{T}_{2}$-weighted Dixon TSE protocol can be performed in a free-breathing mode and leads to acceptable image quality in regions that might be affected by breathing and motion artifacts (eg, the paraspinal muscles or shoulder region). Third, the fat suppression in the water-separated images obtained with the Dixon method is less affected by motion artifacts compared with STIR sequences in body regions affected by breathing motion (eg, the shoulder muscles). Fourth, the water-separated and fat-separated images are simultaneously acquired and therefore coregistered, which makes them ideal for subsequent analysis using automated segmentation and image analysis techniques.
The applied rating scales for fatty and edematous changes are mainly used for clinical assessment of muscle pathologies, such as the definition of disease specific patterns of muscle involvement or direction of muscle biopsies. ${ }^{6,11,12}$ In this clinical setting, the two ratings are mostly used in consensus by two readers. ${ }^{13,14}$ To the best of the author's knowledge, a quantitative evaluation of the interrater accuracy of the ratings scales for edematous changes was performed in the present work for the first time. The observed interrater agreement of the $T_{2}$-weighted images assessing edematous changes was lower compared with the ratings of fatty infiltration. This quantifies the empirical finding that the evaluation of intramuscular hyperintensities in $\mathrm{T}_{2}$-weighted images indicating edematous changes is more difficult and therefore subjective than rating of $T_{1}$-hyperintense fatty changes of muscle tissue. Edematous changes in muscle tissue can be very discrete and $\mathrm{T} 2$ signal alterations can be found in healthy muscle tissue as well, leading to misinterpretation and overestimation. Therefore, a certain degree of expertise in musculoskeletal radiology is necessary for a reliable evaluation of pathological changes in $\mathrm{T}_{2}$-weighted images. In case of requirement of more specific quantitative values for example to monitor disease progression or therapy effectiveness, quantitative imaging techniques such as quantitative chemical shift encoding-based water-fat MRI ${ }^{15-17}$ or T2 mapping ${ }^{18,19}$ should be considered to be used.

The healthy muscle signal in the $\mathrm{T}_{2}$-weighted STIR images is in general lower than the healthy muscle signal in the $\mathrm{T}_{2}$-weighted Dixon TSE water images for the same echo time, owing to inversion recovery-induced muscle signal loss and the fact that the STIR technique will not result in a perfect fat suppression because of the different $\mathrm{T} 1$ relaxation times of the multiple fat peaks. Therefore, signal of healthy muscle tissue is lower in $\mathrm{T}_{2}$-weighted STIR images compared with signal of healthy muscle tissue in T2-weighted Dixon TSE water images, whereas suppressed fat signal in $\mathrm{T}_{2}$-weighted STIR images is still higher than the signal of fatty tissues in $\mathrm{T}_{2}$-weighted Dixon TSE water images. Thus, the relative signal difference between healthy muscle tissue and fatty tissue in $\mathrm{T}_{2}$-weighted STIR images is less prominent than the relative signal difference between healthy muscle tissue and fatty tissue in water images of the $\mathrm{T}_{2}$-weighted Dixon TSE sequence. The higher relative signal difference between healthy
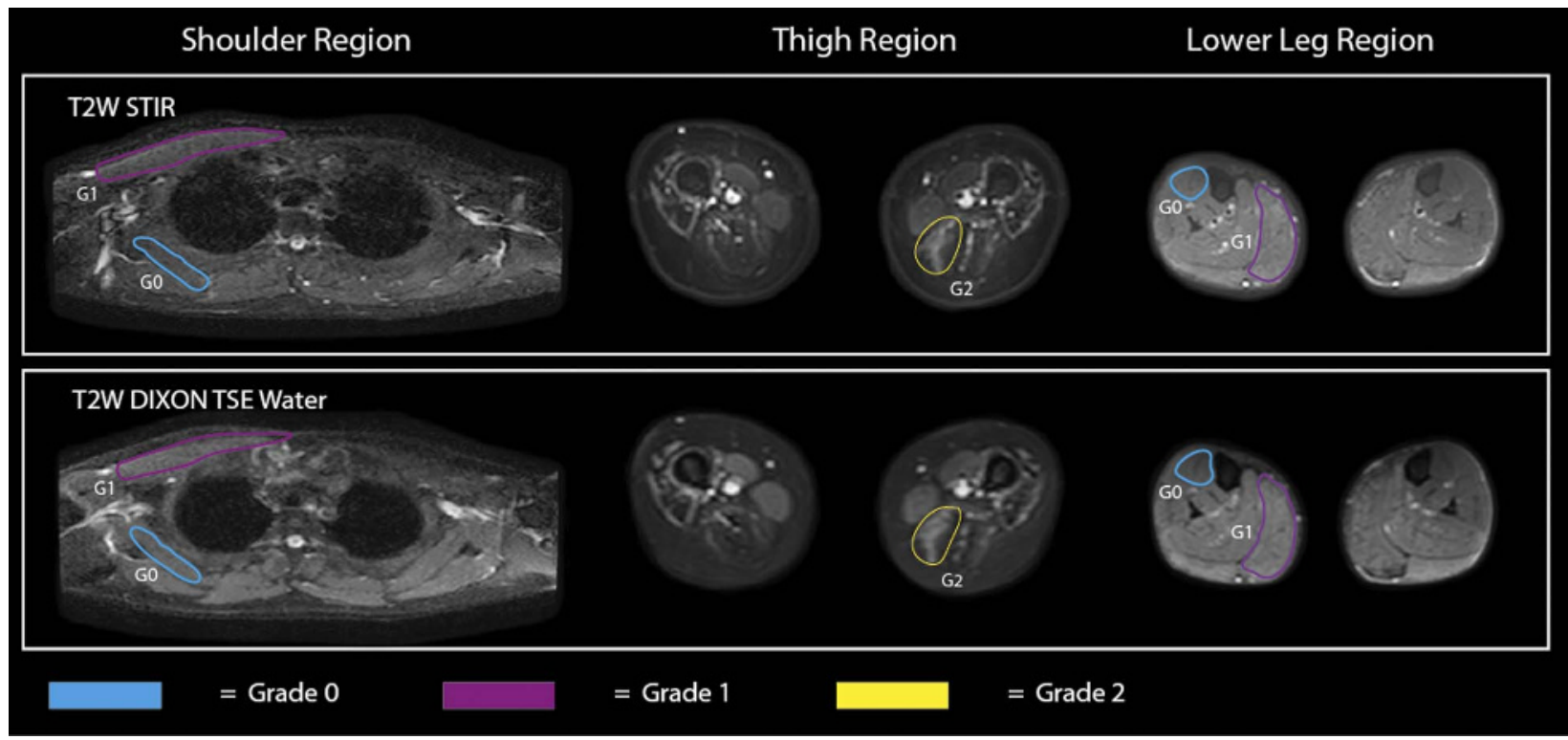

FIGURE 3. Morrow grading of edema based on $\mathrm{T}_{2}$-weighted STIR and $\mathrm{T}_{2}$-weighted Dixon TSE water images in a patient's shoulder region (DM2), a patient's thigh region (BMD), and a patient's lower leg region (CNM). Figure 3 can be viewed online in color at www.jcat.org. 
muscle tissue and fatty tissue in the $\mathrm{T}_{2}$-weighted Dixon TSE sequence might lead to a visual overestimation of the contrast of healthy muscle tissue which could be interpreted as intramuscular hyperintensity indication edema. Therefore, a careful comparison of the single muscles within one patient is necessary to evaluate hyperintensities correctly and expertise in musculoskeletal radiology is required. Another possible way to deal with the higher muscle signal in the $\mathrm{T}_{2}$-weighted Dixon TSE would be a lengthening of the echo time to reduce the strong contrast between healthy muscle and fat tissue. Apart from that, the higher SNR of the $\mathrm{T}_{2}$-weighted Dixon TSE could be used to further reduce scan time.

To the best of the authors' knowledge, there has not yet been a study correlating water $\mathrm{T} 2$ elevation in muscle tissue with histological findings comparable to the work by Zanetti et $\mathrm{al}^{20}$ on edematous changes in bone marrow. Muscle edema remains a rather unspecific finding representing water accumulation after exercise, muscle denervation, inflammation, infection, trauma, treatmentrelated conditions, rhabdomyolysis, or disease activity in inherited myopathies/muscle dystrophies. ${ }^{3,18,21}$ The appearance of the $\mathrm{T}_{2}$-weighted signal changes in the water $\mathrm{T}_{2}$-weighted Dixon TSE images can be different from the $\mathrm{T}_{2}$-weighted STIR images owing to the lack of $\mathrm{T}_{1}$ recovery effects in Dixon TSE. Nevertheless, the present work shows that the radiological diagnosis based on $\mathrm{T}_{2}$-weighted STIR and $\mathrm{T}_{2}$-weighted Dixon TSE water images is mainly identical, although it remains unclear what is the histological correlation to the water T2 elevation in the individual case and thus which sequence is showing the rather real pathology. The fact that water T2 elevation in muscle tissue has not yet been correlated with biopsy results could be seen as a limitation of the study, which should be object of further research.

The present study has some additional limitations. First, fatseparated $\mathrm{T}_{1}$-weighted Dixon FFE images were used for the grading of fatty infiltration instead of traditional $\mathrm{T}_{1}$-weighted TSE images. Grading of fatty infiltration was assessed with $\mathrm{T}_{1}$-weighted FFE because images obtained with this method can be acquired faster than $\mathrm{T}_{1}$-weighted TSE images and because fat-separated $\mathrm{T}_{1}$-weighted Dixon images can be more easily compared with fat-separated $\mathrm{T}_{2}$-weighted Dixon images. Second, only 24 and not all body muscles were graded for fatty infiltration and edematous changes. Most of the rated muscles were located in the thigh region, which is the most typical region affected in patients with NMDs. Thus, a certain bias of the results might be possible.

\section{CONCLUSIONS}

The present study has shown that $\mathrm{T}_{2}$-weighted Dixon TSE acquisition can be used for accelerated simultaneous grading of whole-body skeletal muscle fatty infiltration and edema in patients with NMDs in replacement of the traditional sequential imaging using separate $T_{1}$-weighted acquisitions and $T_{2}$-weighted STIR acquisitions.

\section{ACKNOWLEDGMENT}

The present work was supported by the German Society for Muscle Diseases and Philips Healthcare.

\section{REFERENCES}

1. Carlier PG, Azzabou N, de Sousa PL, et al. Skeletal muscle quantitative nuclear magnetic resonance imaging follow-up of adult Pompe patients. $J$ Inherit Metab Dis. 2015;38:565-572.

2. Forbes SC, Willcocks RJ, Triplett WT, et al. Magnetic resonance imaging and spectroscopy assessment of lower extremity skeletal muscles in boys with Duchenne muscular dystrophy: a multicenter cross sectional study. PLoS One. 2014;9:e106435.

3. Kim HK, Laor T, Horn PS, et al. T2 mapping in Duchenne muscular dystrophy: distribution of disease activity and correlation with clinical assessments. Radiology. 2010;255:899-908.

4. Hollingsworth KG, de Sousa PL, Straub V, et al. Towards harmonization of protocols for MRI outcome measures in skeletal muscle studies: consensus recommendations from two TREAT-NMD NMR workshops, 2 May 2010, Stockholm, Sweden, 1-2 October 2009, Paris, France. Neuromuscul Disord. 2012;22 Suppl 2:S54-S67.

5. Poliachik SL, Friedman SD, Carter GT, et al. Skeletal muscle edema in muscular dystrophy: clinical and diagnostic implications. Phys Med Rehabil Clin N Am. 2012;23:107-122, xi.

6. Mercuri E, Pichiecchio A, Allsop J, et al. Muscle MRI in inherited neuromuscular disorders: past, present, and future. J Magn Reson Imaging. 2007;25:433-440

7. Morrow JM, Matthews E, Raja Rayan DL, et al. Muscle MRI reveals distinct abnormalities in genetically proven non-dystrophic myotonias. Neuromuscul Disord. 2013;23:637-646.

8. Eggers H, Brendel B, Duijndam A, et al. Dual-echo Dixon imaging with flexible choice of echo times. Magn Reson Med. 2011;65:96-107.

9. Reeder SB, Pineda AR, Wen Z, et al. Iterative decomposition of water and fat with echo asymmetry and least-squares estimation (IDEAL): application with fast spin-echo imaging. Magn Reson Med. 2005;54: 636-644

10. Jakobsson U, Westergren A. Statistical methods for assessing agreement for ordinal data. Scand J Caring Sci. 2005;19:427-431.

11. Wattjes MP, Kley RA, Fischer D. Neuromuscular imaging in inherited muscle diseases. Eur Radiol. 2010;20:2447-2460.

12. May DA, Disler DG, Jones EA, et al. Abnormal signal intensity in skeletal muscle at MR imaging: patterns, pearls, and pitfalls. Radiographics. 2000; 20 Spec No:S295-S315.

13. Kornblum C, Lutterbey G, Bogdanow M, et al. Distinct neuromuscular phenotypes in myotonic dystrophy types 1 and $2:$ a whole body highfield MRI study. J Neurol. 2006;253:753-761.

14. Peric S, Maksimovic R, Banko B, et al. Magnetic resonance imaging of leg muscles in patients with myotonic dystrophies. J Neurol. 2017;264: 1899-1908.

15. Karampinos DC, Yu H, Shimakawa A, et al. $\mathrm{T}_{1}$-corrected fat quantification using chemical shift-based water/fat separation: application to skeletal muscle. Magn Reson Med. 2011;66:1312-1326.

16. Reeder SB, Hu HH, Sirlin CB. Proton density fat-fraction: a standardized MR-based biomarker of tissue fat concentration. J Magn Reson Imaging. 2012;36:1011-1014.

17. Burakiewicz J, Sinclair CDJ, Fischer D, et al. Quantifying fat replacement of muscle by quantitative MRI in muscular dystrophy. J Neurol. 2017;264: 2053-2067.

18. Marty B, Baudin PY, Reyngoudt H, et al. Simultaneous muscle water T2 and fat fraction mapping using transverse relaxometry with stimulated echo compensation. NMR Biomed. 2016;29:431-443.

19. Klupp E, Weidlich D, Schlaeger S, et al. B1-insensitive T2 mapping of healthy thigh muscles using a T2-prepared 3D TSE sequence. PLoS One. 2017;12:e0171337.

20. Zanetti M, Bruder E, Romero J, et al. Bone marrow edema pattern in osteoarthritic knees: correlation between MR imaging and histologic findings. Radiology. 2000;215:835-840.

21. Mike P, Wattjes DF. Neuromuscular Imaging. New York, NY: Springer Science + Business Media; 2013. 